Göteborg-ITP-96-14

CTP-TAMU-59/96

hep-th/9611159

November, 1996

\title{
The Dirichlet Super- $p$-Branes in Ten-Dimensional Type IIA and IIB Supergravity
}

\author{
Martin Cederwall $^{a}$, Alexander von Gussich ${ }^{a}$, Bengt E.W. Nilsson ${ }^{a, b}$, \\ Per Sundell $^{b}$ and Anders Westerberg ${ }^{a}$ \\ ${ }^{a}$ Institute of Theoretical Physics \\ Göteborg University and Chalmers University of Technology \\ S-412 96 Göteborg, Sweden \\ tfemc, tfeavg, tfebn, tfeawg@fy.chalmers.se \\ ${ }^{b}$ Center for Theoretical Physics \\ Texas A \& $M$ University \\ College Station, Texas 77843, USA \\ per@chaos . tamu .edu
}

\begin{abstract}
We give the full supersymmetric and $\kappa$-symmetric actions for the Dirichlet $p$-branes, including their coupling to background superfields of ten-dimensional type IIA and IIB supergravity.
\end{abstract}


Cederwall, von Gussich, Nilsson, Sundell, Westerberg: "The Dirichlet Super- $p$-Branes $\ldots . \ldots$

\section{INTRODUCTION}

In a previous paper [1] the $\kappa$-symmetric Dirichlet three-brane action including its coupling to a general type ПВ on-shell supergravity background was constructed, and some key aspects for general $\mathrm{D} p$-branes were anticipated. Using essentially the same methods we here complete this construction by deriving all the $\kappa$-symmetric Dirichlet $p$-brane actions including their on-shell background couplings. For even and odd $p$ this background is type IIA and type IB supergravity, respectively. The subject of supersymmetric D-branes has also been addressed in ref. [2], where the form of the $\kappa$-transformations was conjectured for general $p$ in a flat background.

Given the important rôle played by D-branes in non-perturbative string theory [3,4,5], it is an urgent issue to obtain a fuller understanding of their inherent dynamics. Progress in this area would also mean filling a gap in our present picture of the theory of extended objects $[6,7,8,9,10]$, where Dbranes generalize ordinary $p$-branes by containing non-scalar world-volume fields. We also believe that such a detailed understanding of the mechanisms at work will turn out to be valuable when one addresses aspects of a more fundamental underlying theory, M-theory $[11,12,13,14,15]$. The low-energy limit of M-theory, eleven-dimensional supergravity [16,17], contains extended objects, namely a membrane and a five-brane $[18,19]$, and we envisage that the analysis of these, and of the various string dualities $[11,12,13,20-28]$ (hopefully) explained by M-theory, will benefit from the techniques presented in this paper. We will comment further on this in the last section.

We start out by reviewing the bosonic D-brane actions in section 2. Section 3 discusses the supergravity backgrounds in which the D-branes propagate, and introduces the algebraic constraints necessary for the supergravity theories (thereby put on-shell) and, as it turns out, also for the supersymmetry of the D-brane actions. In the presentation of the result in section 4 we treat the type ПIA and type ПВ branes in parallel, in order to stress the similarities as far as the $\kappa$-symmetry is concerned. Here we verify that, given the constraints of section 3 on the fields in the NS-NS sector (coupling to the kinetic term of the D-brane action) the constraints in the RR sector (entering a Wess-Zumino term) may be read off from $\kappa$-symmetry. Thus, consistent propagation of D-branes demands a background solving the equations of motion of the appropriate supergravity theory. In section 5, we solve the Bianchi identities relevant to our discussion, and find that the solutions agree with the constraints given in section 3 . This is the only instance where essential differences between the IIA and IB cases emerge. In section 6 , we try to put the results of this paper in a somewhat broader perspective, and comment on the possible applicability of our techniques and of the mechanisms at work in the D-brane actions for some unsolved related problems. The paper ends by three appendices. In appendix A we describe our conventions and notation. Appendix B treats the properties of the projection matrix associated with $\kappa$-symmetry, and finally, in appendix $\mathrm{C}$ we present the details of the proof of $\kappa$-symmetry that we left out in section 4 .

\section{Preliminaries}

A bosonic $\mathrm{D} p$-brane is described by a target space and world-volume covariant action $I=I_{\mathrm{DBI}}+I_{\mathrm{WZ}}$. 
The first term is the Dirac-Born-Infeld action [29]

$$
I_{\mathrm{DBI}}=\int_{M} d^{p+1} \xi \mathscr{L}_{\mathrm{DBI}}
$$

where

$$
\begin{aligned}
\mathscr{L}_{\mathrm{DBI}} & =-e^{-\phi} \sqrt{-\operatorname{det}\left(e^{\frac{1}{2} \phi} g_{i j}+\left(F_{i j}-B_{i j}\right)\right)} \\
& =-e^{\frac{p-3}{4} \phi} \sqrt{-\operatorname{det}\left(g_{i j}+e^{-\frac{1}{2} \phi}\left(F_{i j}-B_{i j}\right)\right)} .
\end{aligned}
$$

Here $\xi^{i}$ are the coordinates of the $(p+1)$-dimensional bosonic world-volume $M$, which is mapped by world-volume fields $X^{m}$ into ten-dimensional curved target space with vielbein $e_{m}{ }^{a}$. This embedding induces a world-volume metric $g_{i j}=e_{i}{ }^{a} e_{j}{ }^{b} \eta_{a b}$, where $e_{i}{ }^{a}=\partial_{i} X^{m} e_{m}{ }^{a}$ is the pullback of the target space vielbein to the world-volume. The world-volume also carries an intrinsic abelian gauge field $A$ with field strength $F=d A=\frac{1}{2} d \xi^{j} \wedge d \xi^{i} F_{i j} .{ }^{\star}$ A bosonic D $p$-brane thus has $(10-p-1)+(p-1)=8$ degrees of freedom. Furthermore, $B_{i j}$ and $\phi$ are the pullbacks of the target space Neveu-Schwarz-Neveu-Schwarz (NS-NS) two-form and dilaton fields, respectively. The exponential of the dilaton defines the coupling of the theory. The dilaton dependence in (2.2) corresponds to an Einstein frame metric. Note that for $p=3$ the dilaton coupling affects only $\mathscr{F} \equiv F-B$. This is related to the fact that the $\mathrm{D} 3$-brane is self-dual under the $\mathrm{SL}(2 ; \mathbb{Z})$ of type IIB $\left[3^{0}, 3^{1}\right]$. We will elaborate further on the rôles of the various fields appearing in the action above in the following sections.

The second part of the action is the Wess-Zumino term [32,33], containing the couplings of the $\mathrm{D} p$-brane to the target space Ramond-Ramond (RR) fields. It can be written compactly as

$$
I_{\mathrm{wz}}=\int_{M} e^{\mathscr{F}} \wedge C,
$$

where the RR $n$-form gauge potentials (pulled back to the world-volume) are collected in

$$
C=\bigoplus_{n} C_{(n)}
$$

The integration in (2.3) automatically selects the proper forms in the sum (2.4) - odd for type ПI and even for type ППВ.

We now wish to construct the corresponding target superspace covariant and $\kappa$-symmetric $\mathrm{D} p$-brane action (both local symmetries). The target superspace coordinates $Z^{M}$ are formed out of the 10 bosonic $X^{m}$ and of 32 fermionic coordinates $\theta^{\mu}$ grouped into two Majorana-Weyl spinors. In type IIA superspace these two spinors carry opposite chiralities, while in type IIB superspace they are of the same chirality which is conventionally chosen to be positive. The implementation of manifest target superspace covariance for the action is rather straightforward; the prerequisites

* Here we have omitted the factor $\frac{\alpha^{\prime}}{2 \pi}$ in front of $F$. We have also left out the world-volume tension appearing in the DBI action. 
for this step will be presented in detail in the next section. The world-volume $M$, which is still bosonic, is then mapped into the target superspace by fields $Z^{M}(\xi)$. The resulting D $p$-brane theory will therefore seemingly have 16 fermionic on-shell degrees of freedom in the world-volume. The rôle of the $\kappa$-symmetry to be treated in section 4 is to reduce this number by half.

\section{The $D=10$ SUPERGRAVITy BACKGround}

The curved target superspace geometry is described by the vielbein one-form $E^{A}=d Z^{M} E_{M}{ }^{A}$ and the torsion two-form

$$
T^{A}=D E^{A} \equiv d E^{A}+E^{B} \wedge \omega_{B}{ }^{A} .
$$

The covariant derivative $D$ is defined in type IIA superspace using a Lorentz connection one-form $\omega_{A}^{B}$. Note that $d$ has a right action; see appendix A. In the case of type IB, there is in addition a $\mathrm{U}(1)$ connection associated with the fact that the scalars of type IIB supergravity live in the coset space $\mathrm{SU}(1,1) / \mathrm{U}(1)$ (see e.g. ref. [34]). The Lorentzian assumption amounts to the conditions $\omega_{a}{ }^{\beta}=0=\omega_{\alpha}{ }^{b}$. The Lorentzian field strength, i.e. the curvature two-form, is defined as

$$
R_{A}^{B}=d \omega_{A}^{B}+\omega_{A}^{C} \wedge \omega_{C}^{B},
$$

which by the Lorentzian assumption obeys

$$
R_{a}{ }^{\beta}=0=R_{\alpha}{ }^{b}
$$

We also have the "first" and "second" Bianchi identities:

$$
\begin{aligned}
D T^{A} & =E^{B} \wedge R_{B}{ }^{A}, \\
D R_{A}{ }^{B} & =0 .
\end{aligned}
$$

This structure alone cannot yield a consistent on-shell supersymmetric target space background; there is need for more on-shell bosonic degrees of freedom to balance the excess of fermions in the above field content after it has been constrained in the fashion explained below. The additional on-shell bosons required are supplied by the abelian super-field strengths - the NS-NS superthree-form

$$
H_{(3)}=d B_{(2)}
$$

and the RR super- $n$-forms *

$$
\begin{gathered}
R=e^{B_{(2)}} \wedge d\left(e^{-B_{(2)}} \wedge C\right) \equiv \bigoplus_{n=1}^{10} R_{(n)}, \\
C \equiv \bigoplus_{n=0}^{9} C_{(n)} .
\end{gathered}
$$

* We will not treat the case of non-vanishing cosmological constant in type IIA massive supergravity, i.e., $R_{(0)}=m \neq 0$. 
Cederwall, von Gussich, Nilsson, Sundell, Westerberg: “The Dirichlet Super-p-Branes ...” ....

These field strengths obey the Bianchi identities

$$
\begin{array}{r}
d H_{(3)}=0, \\
e^{B_{(2)} \wedge d\left(e^{-B_{(2)}} \wedge R\right)=d R-R \wedge H}=0,
\end{array}
$$

and they are invariant under the gauge transformations

$$
\begin{aligned}
\delta B_{(2)} & =d \lambda, \\
\delta C & =e^{B_{(2)}} \wedge d \mu .
\end{aligned}
$$

Note that the Bianchi identity (3.7) allows one to nullify all even or odd forms in $R$. The onshell type $\Pi \mathrm{A}[35]$ and $\Pi B$ [34] supergravities are then found by truncating to even and odd $R$, respectively, and then imposing the following constraints on the field strength components of canonical dimension less than or equal to $\frac{1}{2}$ :

$$
T_{\alpha \beta}{ }^{c}=2 i \gamma_{\alpha \beta}^{c}, \quad T_{a \beta}{ }^{c}=0,
$$

ПА: $\quad T_{\alpha \beta}{ }^{\gamma}=\frac{3}{2} \delta_{(\alpha}{ }^{\gamma} \Lambda_{\beta)}+2\left(\gamma_{11}\right)_{(\alpha}{ }^{\gamma}\left(\gamma_{11} \Lambda\right)_{\beta)}-\frac{1}{2}\left(\gamma_{a}\right)_{\alpha \beta}\left(\gamma^{a} \Lambda\right)^{\gamma}$

$$
+\left(\gamma_{a} \gamma_{11}\right)_{\alpha \beta}\left(\gamma^{a} \gamma_{11} \Lambda\right)^{\gamma}+\frac{1}{4}\left(\gamma_{a b}\right)_{(\alpha}{ }^{\gamma}\left(\gamma^{a b} \Lambda\right)_{\beta)} \text {, }
$$

IIB: $\quad T_{\alpha \beta}^{\gamma}=-(J)_{\left(\alpha^{\gamma}\right.}^{\gamma}(J \Lambda)_{\beta)}+(K)_{(\alpha}^{\gamma}(K \Lambda)_{\beta)}$

$$
+\frac{1}{2}\left(\gamma_{a} J\right)_{\alpha \beta}\left(\gamma^{a} J \Lambda\right)^{\gamma}-\frac{1}{2}\left(\gamma_{a} K\right)_{\alpha \beta}\left(\gamma^{a} K \Lambda\right)^{\gamma},
$$

$H_{\alpha \beta \gamma}=0$,

ПА: $\quad H_{a \beta \gamma}=-2 i e^{\frac{1}{2} \phi}\left(\gamma_{11} \gamma_{a}\right)_{\beta \gamma}$,

$H_{a b \gamma}=e^{\frac{1}{2} \phi}\left(\gamma_{a b} \gamma_{11} \Lambda\right)_{\gamma}$,

IIB: $\quad H_{a \beta \gamma}=-2 i e^{\frac{1}{2} \phi}\left(K \gamma_{a}\right)_{\beta \gamma}$,

$H_{a b \gamma}=e^{\frac{1}{2} \phi}\left(\gamma_{a b} K \Lambda\right)_{\gamma}$,

$R_{(n) \alpha \beta \gamma A_{1} \ldots A_{n-3}}=0$,

IIA: $\quad R_{(n) a_{1} \ldots a_{n-2} \alpha \beta}=2 i e^{\frac{n-5}{4} \phi}\left(\gamma_{a_{1} \ldots a_{n-2}}\left(\gamma_{11}\right)^{\frac{n}{2}}\right)_{\alpha \beta}$,

$R_{(n) a_{1} \ldots a_{n-1} \alpha}=-\frac{n-5}{2} e^{\frac{n-5}{4} \phi}\left(\gamma_{a_{1} \ldots a_{n-1}}\left(-\gamma_{11}\right)^{\frac{n}{2}} \Lambda\right)_{\alpha}$,

IIB: $\quad R_{(n) a_{1} \ldots a_{n-2} \alpha \beta}=2 i e^{\frac{n-5}{4} \phi}\left(\gamma_{a_{1} \ldots a_{n-2}} K^{\frac{n-1}{2}} I\right)_{\alpha \beta}$,

$R_{(n) a_{1} \ldots a_{n-1} \alpha}=-\frac{n-5}{2} e^{\frac{n-5}{4} \phi}\left(\gamma_{a_{1} \ldots a_{n-1}} K^{\frac{n-1}{2}} I \Lambda\right)_{\alpha}$.

$\Lambda_{\alpha}=\frac{1}{2} \partial_{\alpha} \phi$ 
Here $K$ and $J$ are $\mathrm{SO}(2)$ matrices appearing in the real formulation of type IIB supergravity; see appendix A for further discussion and an explanation of our conventions for the $\gamma$-matrices. We have included here the constraint relating the dimension-0 scalar superfield $\phi$ containing the target space dilaton to the superfield $\Lambda_{\alpha}$ whose leading component is the spinor of the appropriate supergravity multiplet.

There is of course some arbitrariness in the choice of constraints. The numerical coefficient in front of the dimension- 0 component of the torsion is free to choose, but then the normalization of the field strength in the Dirac-Born-Infeld part of the action will fix the absolute value of the one in front of the dimension- 0 component of $H$, and also relate the ones for the $R$ 's to the normalization of the Wess-Zumino term. Once these are chosen, and the relation between $\phi$ and $\Lambda$ is fixed, no freedom remains. Our conventions for $H$ differ by a sign from those in ref. [1], which will also imply sign differences in the projection matrix for the parameter of $\kappa$-symmetry.

The virtue of these constraints is that the Bianchi identities (3.7) and (3.4) and the Lorentzian assumption (3.3) now turn from identities into equations for the component fields of dimension 0 and higher, thus reducing the enormous unconstrained field content precisely down to the field content of the on-shell type IIA and type IIB super-multiplets respectively. Of course, one in particular has to check that these equations are solved at dimensions 0 and $\frac{1}{2}$ by (3.9). This will be done in section 5. A more general analysis would reveal that the "higher" RR field strengths $R_{(n)}$ with $n \geq 5$ are auxiliary in the sense that their propagating degrees of freedom which sit in the unconstrained dimension- 1 components $R_{(n) a_{1} \ldots a_{n}}$ are related by Hodge duality to the propagating degrees of freedom of the "lower" RR field strengths $R_{(10-n)}$ contained in analogous bosonic $(10-n)$-forms $r_{(10-n)}[32,9]$. In fact, in an on-shell background as we have here, one can consistently incorporate both a potential and its dual potential simultaneously, since the Bianchi identities and the field equations in such a situation are on equal footing.

To complete the picture of the target space background we also include the auxiliary dual NS-NS field strengths

$$
\begin{array}{ll}
\text { IIA: } \quad H_{(7)}=d B_{(6)}-\frac{1}{2} C_{(1)} \wedge R_{(6)}+\frac{1}{2} C_{(3)} \wedge R_{(4)}-\frac{1}{2} C_{(5)} \wedge R_{(2)}, \\
\text { IВ: } \quad H_{(7)}=d B_{(6)}+\frac{1}{2} C_{(0)} \wedge R_{(7)}-\frac{1}{2} C_{(2)} \wedge R_{(5)}+\frac{1}{2} C_{(4)} \wedge R_{(3)}-\frac{1}{2} C_{(6)} \wedge R_{(1)},
\end{array}
$$

which are subject to the constraints

$$
\begin{array}{ll}
\text { IIA: } & H_{a_{1} \ldots a_{5} \alpha \beta}=2 i e^{-\frac{\phi}{2}}\left(\gamma_{a_{1} \ldots a_{5}}\right)_{\alpha \beta}, \\
& H_{a_{1} \ldots a_{6} \alpha}=-e^{-\frac{\phi}{2}}\left(\gamma_{a_{1} \ldots a_{6}} \Lambda\right)_{\alpha}, \\
\text { IB }: & H_{a_{1} \ldots a_{5} \alpha \beta}=2 i e^{-\frac{\phi}{2}}\left(\gamma_{a_{1} \ldots a_{5}} K\right)_{\alpha \beta}, \\
& H_{a_{1} \ldots a_{6} \alpha}=-e^{-\frac{\phi}{2}}\left(\gamma_{a_{1} \ldots a_{6}} K \Lambda\right)_{\alpha},
\end{array}
$$


and obey the Bianchi identities

$$
\begin{array}{ll}
\text { IA: } & d H_{(7)}+R_{(2)} \wedge R_{(6)}-\frac{1}{2} R_{(4)} \wedge R_{(4)}, \\
\text { ПВ: } \quad d H_{(7)}+R_{(1)} \wedge R_{(7)}-R_{(3)} \wedge R_{(5)}=0 .
\end{array}
$$

Though not appearing explicitly in the Bianchi identities (3.7), this field strength plays a rôle in the solution of the Bianchi identities for type IB, as will be explained in more detail in section 5 .

Whereas the auxiliary field content has previously been seen as an extravagance from a target space point of view, its crucial function in the construction of $\kappa$-symmetric $p$-brane actions is now well understood. There is only one exception, namely the auxiliary NS-NS potential $B_{(6)}$ which does not enter the construction of any of the $p$-brane actions that we know explicitly. The potential rôle for $B_{(6)}$ in the construction of ( $\kappa$-symmetric) world-volume actions will be discussed briefly in section 6 .

\section{The Actions}

As a consequence of the embedding of the bosonic world-volume $M$ into target superspace, all target space structures are pulled back to $M^{\star}$. The pull-back of the super-vielbein is given by

$$
E^{A} \equiv d \xi^{i} E_{i}{ }^{A}=d \xi^{i} \partial_{i} Z^{M} E_{M}{ }^{A}
$$

Consequently, the pull-back of a super- $n$-form $\Omega$ has the components

$$
\Omega_{i_{1} \ldots i_{n}}=E_{i_{n}}^{A_{n}} \cdots E_{i_{1}}^{A_{1}} \Omega_{A_{1} \ldots A_{n}} .
$$

The pull-back of the Lorentz metric is given by

$$
g_{i j}=E_{i}^{a} E_{j}^{b} \eta_{a b}
$$

By insisting on manifest target space gauge invariance, one is led to introduce the two-form field strength

$$
\mathscr{F}=F-B_{(2)},
$$

which is invariant under the target space transformation (3.8) combined with $\delta A=\lambda$. We also note the gauge invariance of the Wess-Zumino term:

$$
\delta\left(e^{\mathscr{F}} \wedge C\right)=e^{\mathscr{F}} \wedge e^{B_{(2)}} \wedge d \mu=e^{d A} \wedge d \mu=d\left(e^{d A} \mu\right) .
$$

\footnotetext{
* We follow the convention to use the same notation for the super-forms in target space as for their pull-backs to the world-volume (where they become ordinary bosonic forms).
} 
Using this requisite we can now write the following manifestly target superspace covariant and gauge invariant as well as world-volume covariant generalization of the action given in section 2 :

$$
I=I_{\mathrm{DBI}}+I_{\mathrm{WZ}}=-\int_{M} d^{p+1} \xi e^{\frac{p-3}{4} \phi} \sqrt{-\operatorname{det}\left(g_{i j}+e^{-\frac{1}{2} \phi} \mathscr{F}_{i j}\right)}+\int_{M} e^{\mathscr{F}} \wedge C .
$$

Formally, this action looks identical to the one in $(2.1)-(2.3)$. The crucial difference is of course that the bosonic background fields have been replaced by their corresponding superfields.

Remarkably enough, the action (4.6) is now invariant under the following local variation

$$
\begin{aligned}
\delta_{\kappa} Z^{M} & =\kappa^{A} E_{A}{ }^{M}, \quad \kappa^{a}=0, \\
\delta_{\kappa} A & =i_{\kappa} B_{(2)},
\end{aligned}
$$

provided that the spinorial variational parameter $\kappa$ obeys

$$
\Gamma \kappa=\kappa .
$$

Here the matrix $\Gamma$ (acting in spinor space) is given by

$$
d^{p+1} \xi \Gamma=-\left.\frac{e^{\frac{1}{4}(p-3) \phi}}{\mathscr{L}_{\mathrm{DBI}}} \exp \left(e^{-\frac{1}{2} \phi} \mathscr{F}\right) \wedge X Y\right|_{\text {vol }}
$$

with

$$
X=\bigoplus_{n} \gamma_{(2 n+q)} P^{n+q}
$$

and

$$
\begin{array}{llll}
\text { ПА: } & P=\gamma_{11}, & Y=\mathbb{1}, & q=1, \\
\text { ПВ: } & P=K, & Y=I, & q=0 .
\end{array}
$$

Here we have used the notation $\gamma_{(n)}=\frac{1}{n !} d \xi^{i_{n}} \wedge \ldots \wedge d \xi^{i_{1}} \gamma_{i_{1} \ldots i_{n}}$, where $\gamma_{i_{1} \ldots i_{n}}$ is the pullback of the corresponding target space Dirac $\gamma$-matrix. For further explanation of the notation, we again refer to appendix A. This expression for $\Gamma$ was conjectured for the $\Pi$ B case in ref. [1].

The construction of the matrix $\Gamma$ is one of the key steps in establishing $\kappa$-symmetry of the $\mathrm{D} p$-brane actions. The crucial properties of $\Gamma$ is that it squares to $\mathbb{1}$ and that the projection matrix $\frac{1}{2}(\mathbb{1}+\Gamma)$ has rank 16 , i.e. half the maximal value. The latter result follows immediately from the observation that $\Gamma$ is traceless and has eigenvalues \pm 1 . For a proof of the property $\Gamma^{2}=\mathbb{1}$, we refer the reader to appendix $B$.

Hence, the $\kappa$-symmetry reduces the number of physical (real) fermionic degrees of freedom from 16 to 8, as required by supersymmetry. Of course, this presupposes that fixing the worldvolume diffeomorphism and gauge invariances turns the target space spinors to spinors also on the 
world-volume, as happens for the superstring and supermembrane. We have thus constructed the supersymmetric Dirichlet $p$-branes for all possible values of $p$ in type ПА and type ПВ supergravity.

Equipped with the matrix $\Gamma$, we are now ready to establish the $\kappa$-invariance of the action (4.6). The basic $\kappa$-variations of the world-volume fields given in (4.7) imply

$$
\begin{aligned}
\delta_{\kappa} g_{i j} & =2 E_{(i}{ }^{a} E_{j)}{ }^{B} \kappa^{\alpha} T_{\alpha B a}, \\
\delta_{\kappa} \mathscr{F}_{i j} & =-E_{j}{ }^{B} E_{i}{ }^{A} \kappa^{\alpha} H_{\alpha A B}, \\
\delta_{\kappa} \phi & =\kappa^{\alpha} \partial_{\alpha} \phi .
\end{aligned}
$$

The expression for $\delta_{\kappa} \mathscr{F}_{i j}$ was obtained using the fact that

$$
\delta_{\kappa} \Omega=\left\{d, i_{\kappa}\right\} \Omega
$$

when $\Omega$ is the pull-back of a super-form *. Note that the definition of the $\kappa$-variation of the worldvolume one-form $A$ given in (4.7) is chosen such as to cancel the term $d\left(i_{\kappa} B_{(2)}\right)$ in the $\kappa$-variation of $B_{2}$. The formula (4.13) is of course also very useful when determining the variation of the WZ term in $(4.6)$, which is readily found to be

$$
\delta_{\kappa} I_{\mathrm{wz}}=\int_{M} e^{\mathscr{F}} \wedge i_{\kappa} R
$$

At this point we can notice that since all field strengths, NS-NS as well as RR, always appear multiplied by $i_{\kappa}$, the conditions for $\kappa$-invariance will only constrain the components $R_{(n) A_{1} \ldots A_{n-1} \alpha}$, i.e. the components of dimension less than or equal to $\frac{1}{2}$.

It is less straightforward to obtain the $\kappa$-variation of the DBI action. Using the matrix identity

$$
\delta \operatorname{det} M=\operatorname{det} M \operatorname{tr}\left(M^{-1} \delta M\right),
$$

we find

$$
\delta_{\Gamma \kappa} I_{\mathrm{DBI}}=\int_{M} d^{p+1} \xi \mathscr{L}_{\mathrm{DBI}}\left(\frac{p-3}{4} \delta_{\Gamma \kappa} \phi+\frac{1}{2} \operatorname{tr}\left\{(g+\tilde{\mathscr{F}})^{-1}\left(\delta_{\Gamma \kappa} g+\delta_{\Gamma \kappa} \tilde{\mathscr{F}}\right)\right\}\right)
$$

where we have defined $\tilde{\mathscr{F}} \equiv e^{-\frac{\phi}{2}} \mathscr{F}$. Note here that we use $\Gamma \kappa$ as the variational parameter. This is because when we insert the expression for $\Gamma$ the factor $\mathscr{L}_{\mathrm{DBI}}$ in $(4.16)$ conveniently cancels.

${ }^{\star}$ To be explicit, $\delta_{\kappa} Z^{*} \Omega=Z^{*} \mathscr{L}_{\kappa} \Omega=Z^{*}\left\{d, i_{\kappa}\right\} \Omega$, where $Z$ is the embedding of the world-volume in target superspace and $\mathscr{L}_{\kappa}$ is the target space Lie derivative induced by the local target superspace vector field $\kappa$. We will not bother to write the $Z^{*}$ explicitly; see previous footnote. 
Inserting in (4.12) the constraints for the torsion $T^{A}$, the NS-NS field strength $H_{(3)}$ and the dilaton $\phi$ given in the previous section, we obtain

$$
\begin{aligned}
\delta_{\Gamma \kappa} g_{i j} & =4 i\left(\bar{E}_{(i} \gamma_{j)} \Gamma \kappa\right), \\
\delta_{\Gamma \kappa} \mathscr{F}_{i j} & =(-1)^{q}\left(4 i\left(e^{\frac{1}{2} \phi} \bar{E}_{[i} \gamma_{j]} P \Gamma \kappa\right)+e^{\frac{1}{2} \phi}\left(\bar{\Lambda} \gamma_{i j} P \Gamma \kappa\right)\right), \\
\delta_{\Gamma \kappa} \phi & =2 \bar{\Lambda} \Gamma \kappa,
\end{aligned}
$$

with $P$ and $q$ defined in (4.11). When inserting these expressions in (4.16), it is convenient to consider the $\Lambda$-independent terms and the terms proportional to $\Lambda$ separately. Referring the reader to appendix $\mathrm{C}$ for the details of these somewhat lengthy but nevertheless illuminating calculations, we only state the final results here: the contribution from the $\Lambda$-independent terms is

$$
\delta_{\Gamma \kappa}^{0} I_{\mathrm{DBI}}=\int_{M} e^{\mathscr{F}} \wedge \bigoplus_{n} 2 i e^{\frac{1}{4}(2 n+q-4) \phi}\left(\bar{\kappa} \gamma_{(2 n+q-1)} P^{n+q} Y \wedge E\right)
$$

while the $\Lambda$-dependent ones yield

$$
\delta_{\Gamma \kappa}^{1 / 2} I_{\mathrm{DBI}}=-\int_{M} e^{\mathscr{F}} \wedge \bigoplus_{n} \frac{1}{2}(2 n+q-4) e^{\frac{1}{4}(2 n+q-4) \phi}\left(\bar{\Lambda} \gamma_{(2 n+q)} P^{n+q} Y \kappa\right)
$$

By comparing with (4.14) it is now straightforward to see that these expressions cancel the contributions to $\delta_{\kappa} I_{\mathrm{Wz}}$ coming from the dimension-0 and dimension- $\frac{1}{2}$ field strength components, respectively, precisely when these components satisfy the constraints given in (3.9).

We would like to stress that the result that the $\kappa$-variation of the DBI action turns out to be a sum of differential forms appears to be quite non-trivial. It is even more gratifying to find that $\kappa$-invariance of the $\mathrm{D} p$-brane action is not only possible, but also forces the background fields to obey the appropriate supergravity equations of motion.

As mentioned in the introduction, the above result for the special case $p=3$ was obtained earlier in ref. [1]. For $p=2$, the type $\Pi \mathrm{A}$ membrane action that we have derived here is equivalent to the previously known $\kappa$-symmetric type IIA membrane action, which by world-volume duality is equivalent to the vertical dimensional reduction of the eleven-dimensional supermembrane [36,37]. The latter action comes with an intrinsic world-volume metric $\gamma_{i j}$. Solving the algebraic equations of motion for $\gamma_{i j}$ using the formula for $p=2$ in ref. [38], we immediately recover the supersymmetrized Dirac-Born-Infeld action (4.6) (albeit the rewriting of the $\Gamma$-matrix is more tedious). In fact, the proof of $\kappa$-symmetry of the D3-brane action is not affected by the introduction of auxiliary world-volume fields (the so called 1.5 order formalism). This observation generalizes immediately to the general case presented here. 


\section{Bianchi IDEntities}

Both chiral and non-chiral type II supergravity in ten dimensions have well-known formulations in superspace $[34,35]$. Therefore, it would in principle be sufficient to compare the dimension- 0 and dimension- $\frac{1}{2}$ components read off from $\kappa$-symmetry and presented in eq. (3.9) with the solutions of the Bianchi identities given in these references.

There are however several reasons to perform a separate check. In type $\Pi \mathrm{A}$, the constraints were originally written in a formalism where Majorana spinor indices are divided into dotted and undotted indices, while we have chosen the more compact notation with a single Majorana index $\alpha$ (we refer to appendix A for our spinor conventions). In type IB, it is natural from a supergravity point of view to use a complex formalism, while the D-branes couple in fundamentally different ways to NS-NS and RR fields, so that we are led to use a real formulation. It is even the case that the fields that naturally enter the D-brane actions do not correspond to real and imaginary parts of the same complex field.

One more reason why we actually have to solve the Bianchi identities is that the higher $\mathrm{D} p$ branes couple to higher antisymmetric tensor fields not present in the ordinary formulations of the supergravities. We thus need explicit control over the behavior of the tensor fields of the dual type $\Pi \mathrm{A}$ and $\Pi \mathrm{B}$ supergravities.

We thus turn our attention to the list of constraints (3.9) and the relevant Bianchi identities, which when written out in irreducible Lorentz components read as follows:

$$
\begin{aligned}
& D_{[A} T_{B C)}{ }^{D}+T_{[A B}{ }^{E} T_{|E| C)}{ }^{D}-R_{[A B C)}{ }^{D}=0, \\
& D_{[A} H_{B C D)}+\frac{3}{2} T_{[A B}{ }^{E} H_{|E| C D)}=0, \\
& D_{\left[A_{n+1}\right.} R_{\left.(n) A_{n} \ldots A_{1}\right)}+\frac{1}{2} n T_{\left[A_{n+1} A_{n}\right.}{ }^{B} R_{\left.(n)|B| A_{n-1} \ldots A_{1}\right)} \\
& \quad=\frac{1}{6} n(n-1) R_{(n-2)\left[A_{n+1} \ldots A_{4}\right.} H_{\left.A_{3} A_{2} A_{1}\right)} .
\end{aligned}
$$

Here the brackets [...) denote graded symmetrization of indices. The constraints listed in (3.9) are not independent, since the Bianchi identities are a set of recursive relations stepping upwards in dimension so that components of dimension $d$ are expressed in terms of components of dimension $\mathrm{d}-\frac{1}{2}$ and below. The canonical dimensions of the torsion components and the components of a field strength super- $n$-form $\Omega$ are given by:

$$
\begin{aligned}
\mathrm{d}\left(T_{A B}{ }^{C}\right) & =\mathrm{d}(A)+\mathrm{d}(B)-\mathrm{d}(C), \\
\mathrm{d}\left(\Omega_{A_{1} \ldots A_{n}}\right) & =\mathrm{d}\left(A_{1}\right)+\cdots+\mathrm{d}\left(A_{n}\right)+1-n,
\end{aligned}
$$

where $\mathrm{d}(\alpha)=\mathrm{d}\left(D_{\alpha}\right)=\frac{1}{2}$ and $\mathrm{d}(a)=\mathrm{d}\left(D_{a}\right)=1$. All tensor fields of negative canonical dimension of course have to vanish, while the dimension- 0 components are fixed by Lorentz invariance up to arbitrary super-scalar coefficients. These coefficients are related through the dimension-0 Bianchi 
identities in (5.1) to various powers of one overall (dimensionless) dilaton superfield coupling $e^{-\frac{1}{2} \phi}$. For the torsion there is an additional degree of freedom corresponding to the higher components in the superfield expansion of the local super-Lorentz parameters that allows one to impose a conventional constraint on its dimension- $\frac{1}{2}$ and 1 components. We have chosen the conventional constraints to be $T_{a \beta}{ }^{c}=0=T_{a b}{ }^{c}$. Once the dimension- 0 constraints and the conventional constraint have been imposed, there is nothing else to be done other than to compute the consequences for the higher-dimensional components. The resulting structure is extremely rigid, in that the same components typically get determined over and over again. If one would march on to higher canonical dimensions one would at dimension 1 find the Hodge duality relation mentioned in section 3 . At dimension $\frac{3}{2}$ and 2 one would find the spin- $\frac{3}{2}$ and spin- 1 equations of motion, respectively. The spin-2 equations of motion sit at dimension 3, while the dimension- $\frac{5}{2}$ equations yield closure of the supersymmetry algebra, i.e., express the variation of the spin- 2 field in terms of the spin- $\frac{3}{2}$ field. For our purposes, however, it is sufficient to only solve the Bianchi identities up to the dimension- $\frac{1}{2}$ level, i.e. to check that (3.9) solves the Bianchi identities (5.1) at dimensions 0 and $\frac{1}{2}$. In the type IIA case this procedure is quite straightforward using only the cyclic Fierz identity in appendix A.

For the tensor fields of type $\Pi \mathrm{B}$, there is an additional complication due to the fact that the scalar fields sit in the coset $\mathrm{SL}(2 ; \mathbb{R}) / \mathrm{U}(1)$. There is a $\mathrm{U}(1)$ gauge field present, and different fields carry different $\mathrm{U}(1)$ charge. In our real formalism, this will be seen as an $\mathrm{SO}(2)$ connection mixing the NS-NS and RR three-forms and seven-forms. Also, any object carrying a spinor index will carry an $\mathrm{SO}(2)$ charge. The $\mathrm{SO}(2)$ action on spinor indices can be understood as the adjoint action of the matrix $I$, so that $I$ itself and the identity matrix are invariant, while $J$ and $K$ are rotated into each other. Since the $\mathrm{U}(1)$ connection is a part of an $\mathrm{SL}(2 ; \mathbb{R})$ connection, it is not consistent to set it to zero, even though it is pure gauge; rather one has to solve the Maurer-Cartan equation for $\mathrm{SL}(2 ; \mathbb{R})$. This is done for our real formalism in ref. [1], and once the gauge choice is performed, one can integrate the Maurer-Cartan equations to obtain two real fields, the dilaton and the RR zero-form potential. The gauge choice we perform makes the $\mathrm{U}(1)$ connection proportional to the $\mathrm{RR}$ one-form field strength, as explained in ref. [1].

The three-form and seven-form field strengths that enter the $\mathrm{D} p$-brane actions for odd $p$ do not carry any $\mathrm{U}(1)$ charge, so the covariant derivatives in their Bianchi identities can be replaced by ordinary exterior derivatives. Nevertheless, since the vielbeins stripped off to obtain the dimension-

0 and dimensions- $\frac{1}{2}$ components do carry charge through their spinor indices, it is important to note that the Bianchi identities for these components require inclusion of connection terms. The gauge choice performed is such that the connection enters the Bianchi identities for $R_{(3)}$ and $R_{(7)}$ but not for $H$. All other tensor fields are neutral under $\mathrm{U}(1)$. Once this detail is taken into account the type IIB case is quite straightforward. The main Fierz identity needed is the cyclic identity of Appendix A.

\section{Conclusions And COMments}

It is worth emphasizing again that it is not at all a priori obvious that the $\kappa$-variation of the Dirac-Born-Infeld action can be written as a wedge products of forms, allowing it to be cancelled 
by the variation of a Wess-Zumino term. We have not investigated whether the class of Lagrangians with this property contains more members than the present example, but we are convinced that

a check order by order in the invariants of the matrix $\mathscr{F}_{i j}$ would not leave much room for any deviations from the Dirac-Born-Infeld expression.

We would like to recapitulate what is known about supersymmetric extended objects and their places in supergravity, string theory and M-theory. When the D-brane actions are understood, the only missing cases in string theory are the solitonic type IIA and IIB five-branes, and the heterotic five-brane, which is T-dual to the well-understood type I five-brane. Both of the type II five-branes couple to the NS-NS six-form potential in the Wess-Zumino term, but otherwise they seem to be very dissimilar. The type IIB five-brane is simply obtained from the D5-brane by performing a duality transformation on the background fields (which in particular reverts the sign of the dilaton), and thus couples to a modified RR two-form potential in the kinetic (actually Dirac-Born-Infeld) term. Its construction is thus in practice performed already in this paper. The type IIA fivebrane, on the other hand, will descend from the eleven-dimensional five-brane by vertical dimensional reduction, and couples to the RR three-form potential in the kinetic term. Essentially, the missing information about supersymmetric extended objects (in Minkowski signature) lies in the eleven-dimensional five-brane, which contains a world-volume two-form potential with self-dual field strength, coupling in the kinetic term to the three-form potential of eleven-dimensional supergravity, and a Wess-Zumino coupling to the six-form potential of the dual supergravity [39]. However, for recent progress in finding a formulation using the embedding formalism, see ref. [40]. We are quite optimistic about the possibilities of obtaining further information by applying the techniques developed in the present paper.

Type IIA supergravity is of course closely related to eleven-dimensional supergravity, and it is plausible that all the structures encountered will receive an explanation in terms of M-theory. While the contents of this statement are clear for $p=1,2,4$ and 5 , it is not at all obvious what the rôles of the D0- and D6-branes are. In ten dimensions they are dual to each other (in the sense that they couple to dual field strengths), but from an eleven-dimensional perspective the supergravity theory (or its dual formulation) contains no fields of appropriate rank they could couple to. The possible formulation of M-theory as a composite zero-brane model [15] is suggestive, and may shed light on this puzzle.

\section{ACKNOWLEDGMENTS}

B.E.W.N is grateful to the Center for Theoretical Physics at Texas A\&M University for its kind hospitality. P.S. would like to thank M.J. Duff, H. Lu, C.N. Pope and E. Sezgin for enlightening comments related to this work. 


\section{Appendix A. Superspace Conventions And Fierz Identities}

We work with a mostly positive Lorentz metric $\eta_{a b}=\operatorname{diag}(-1,1, \ldots, 1)$ and Dirac $\gamma$-matrices obeying $\left\{\gamma_{a}, \gamma_{b}\right\}=2 \eta_{a b}$. The unit-normalized $\gamma$-matrices $\gamma_{a_{1} \ldots a_{n}}$ are defined by

$$
\gamma_{a_{1} \ldots a_{n}}=\gamma_{\left[a_{1}\right.} \cdots \gamma_{\left.a_{n}\right]}
$$

where $\left[a_{1} \ldots a_{n}\right]$ implies antisymmetrization with weight 1 . We frequently collect these objects in forms $\gamma_{(n)}$. The chirality matrix $\gamma_{11} \equiv \gamma_{0} \gamma_{1} \cdots \gamma_{9}$ squares to +1 . The indices of a spinor $\psi^{\alpha}$ and a bispinor $M^{\alpha}{ }_{\beta}$ are (e.g. a $\gamma$-matrix) lowered and raised by means of "natural" matrix multiplication with the antisymmetric charge conjugation matrix $C_{\alpha \beta}=-C_{\beta \alpha}$ and its inverse $C^{\alpha \beta} \equiv\left(C^{-1}\right)^{\alpha \beta}$ :

$$
\begin{aligned}
C_{\alpha \beta} C^{\beta \gamma} & =\delta_{\alpha}{ }^{\gamma}, \\
\psi^{\alpha} & =C^{\alpha \beta} \psi_{\beta}, \\
\psi_{\alpha} & =C_{\alpha \beta} \psi^{\beta}, \\
M_{\alpha}{ }^{\beta} & =C_{\alpha \gamma} M^{\gamma}{ }_{\delta} C^{\delta \beta} .
\end{aligned}
$$

We work with real Majorana spinors $\psi$ obeying

$$
\bar{\psi}=\psi^{T} C
$$

These conventions minimize the number of minus-signs from "see-sawing":

$$
\begin{aligned}
\bar{\psi} M \chi & \equiv \bar{\psi}_{\alpha} M_{\beta}^{\alpha} \chi^{\beta}=\psi^{\alpha} M_{\alpha \beta} \chi^{\beta}, \\
M_{\alpha \beta} M^{\prime \beta}{ }_{\gamma} & =M_{\alpha}{ }^{\beta} M^{\prime}{ }_{\beta \gamma} .
\end{aligned}
$$

As is well known, the $\gamma$-matrix $\left(\gamma_{a_{1} \ldots a_{n}}\right)_{\alpha \beta}$ is antisymmetric when $n=0,3,4,7,8$ and symmetric when $n=1,2,5,6,9,10$.

In type IIA superspace the two 16-component Majorana-Weyl spinorial coordinates of opposite chirality form a 32-component Majorana spinor $\theta^{\alpha}, \alpha=1, . ., 32$, acted on (in the Weyl representation) by real block-off-diagonal Dirac $\gamma_{a}$-matrices, whose off-diagonal 16 by 16 blocks are real Majorana $\sigma_{a}$-matrices, and a diagonal $\gamma_{11}$-matrix. A complete set of matrices for the product of two Majorana spinors is $\gamma_{11}, \gamma_{(1)}, \gamma_{(1)} \gamma_{11}, \gamma_{(2)}, \gamma_{(4)} \gamma_{11}$ and $\gamma_{(5)}$ (symmetric) and $C$,

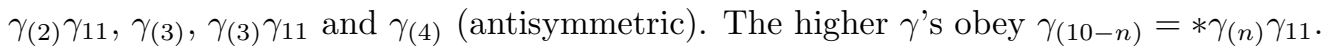

In type IB superspace the two 16-component Majorana-Weyl spinorial coordinates of equal chirality form a 32 -component spinor $\theta^{\alpha}, \alpha=1, . ., 32$, whose $\alpha$-index is a composite index representing the tensor product of a Majorana-Weyl index and an $\mathrm{SO}(2)$ index. This index is acted on by the real Majorana $\sigma_{a}$-matrices, and the real $\mathrm{SO}(2)$ matrices $\mathbb{1}$ and $I, J, K$ defined by $I^{2}=-\mathbb{1}$, $J^{2}=K^{2}=\mathbb{1}, I J=K$ and $I, J, K$ anticommuting. The latter behave as generators for $\operatorname{SL}(2 ; \mathbb{R})$, 
or, equivalently, as the imaginary split quaternionic units (a convenient basis, though not necessary for any calculations, is found in ref. [1]). A complete set of matrices for the tensor product $32 \times 32$ consists of $\gamma_{(1)}, \gamma_{(1)} J, \gamma_{(1)} K, \gamma_{(3)} I, \gamma_{(5)}, \gamma_{(5)} J$ and $\gamma_{(5)} K$ (symmetric) together with $\gamma_{(1)} I, \gamma_{(3)}$, $\gamma_{(3)} J, \gamma_{(3)} K$ and $\gamma_{(5)} I$ (antisymmetric). We have $\gamma_{(10-n)}=* \gamma_{(n)}$.

The basic cyclic Fierz identities are given by:

$$
\begin{array}{ll}
\text { IIA: } \quad\left(\gamma^{a}\right)_{(\alpha \beta}\left(\gamma_{a}\right)_{\gamma) \delta}=-\left(\gamma^{a} \gamma_{11}\right)_{(\alpha \beta}\left(\gamma_{a} \gamma_{11}\right)_{\gamma) \delta}, \\
\text { IIB: } \quad\left(\gamma^{a}\right)_{(\alpha \beta}\left(\gamma_{a}\right)_{\gamma) \delta}=-\left(\gamma^{a} J\right)_{(\alpha \beta}\left(\gamma_{a} J\right)_{\gamma) \delta}=-\left(\gamma^{a} K\right)_{(\alpha \beta}\left(\gamma_{a} K\right)_{\gamma) \delta} .
\end{array}
$$

Repeated use of these identities suffice for a quite direct verification of almost all the Bianchi identities of section 5. Only for the higher type IIB field strengths there seems to be a need for more general Fierz identities. The identity used in the dimension- $\frac{1}{2}$ Bianchi identity for $R_{(7)}$ is

$$
\begin{aligned}
\left(\gamma^{a}\right)_{(\alpha \beta} & \left(\gamma_{a} \gamma_{a_{1} \ldots a_{5}}\right)_{\gamma)} \delta \\
& \left.\left.-\frac{5}{2}\left(\gamma_{a_{1}}\right)_{(\alpha \beta}\left(\gamma_{a_{2} \ldots a_{5}}\right)_{\gamma)}\right)^{\delta}+\frac{5}{2}\left(\gamma_{a_{1}} J\right)_{(\alpha \beta}\left(\gamma_{a_{2} \ldots a_{5}} J\right)_{\gamma)}\right)^{\delta}+\frac{5}{2}\left(\gamma_{a_{1}} K\right)_{(\alpha \beta}\left(\gamma_{a_{2} \ldots a_{5}} K\right)_{\gamma)} \delta \\
& -5\left(\gamma_{a_{1} a_{2} a_{3}} I\right)_{(\alpha \beta}\left(\gamma_{a_{4} a_{5}} I\right)_{\gamma)}{ }^{\delta} \\
& -\frac{1}{2}\left(\gamma_{a_{1} \ldots a_{5}}\right)_{(\alpha \beta} \delta_{\gamma)}{ }^{\delta}+\frac{1}{2}\left(\gamma_{a_{1} \ldots a_{5}} J\right)_{(\alpha \beta} J_{\gamma)} \delta+\frac{1}{2}\left(\gamma_{a_{1} \ldots a_{5}} K\right)_{(\alpha \beta} K_{\gamma)}{ }^{\delta}=0
\end{aligned}
$$

(antisymmetrization in $a_{1} \ldots a_{5}$ is understood), and it can be verified by explicitly tracing with all the symmetric matrices. The Bianchi identity of $R_{(9)}$ involves the Fierz identity

$$
\begin{aligned}
& \left.\left(\gamma^{a}\right)_{(\alpha \beta}\left(\gamma_{a} \gamma_{a_{1} \ldots a_{7}}\right)_{\gamma}\right)^{\delta} \\
& \left.\quad-7\left(\gamma_{a_{1}}\right)_{(\alpha \beta}\left(\gamma_{a_{2} \ldots a_{7}}\right)_{\gamma)}{ }^{\delta}+\frac{7}{4}\left(\gamma_{a_{1}} J\right)_{(\alpha \beta}\left(\gamma_{a_{2} \ldots a_{7}} J\right)_{\gamma)}\right)^{\delta}+\frac{7}{4}\left(\gamma_{a_{1}} K\right)_{(\alpha \beta}\left(\gamma_{a_{2} \ldots a_{7}} K\right)_{\gamma)}{ }^{\delta} \\
& \left.\quad+\frac{21}{4}\left(\gamma_{a_{1} \ldots a_{5}} J\right)_{(\alpha \beta}\left(\gamma_{a_{6} a_{7}} J\right)_{\gamma}\right)^{\delta}+\frac{21}{4}\left(\gamma_{a_{1} \ldots a_{5}} K\right)_{(\alpha \beta}\left(\gamma_{a_{6} a_{7}} K\right)_{\gamma)}{ }^{\delta}-\left(\gamma_{a_{1} \ldots a_{7}} I\right)_{(\alpha \beta} I_{\gamma)}{ }^{\delta}=0
\end{aligned}
$$

We use the superspace conventions in which a super- $n$-form $\Omega_{(n)}$ is expanded as

$$
\Omega_{(n)}=\frac{1}{n !} E^{A_{n}} \wedge \cdots \wedge E^{A_{1}} \Omega_{A_{1} \ldots A_{n}} .
$$

The exterior derivative

$$
d=E^{A} \partial_{A} \equiv d Z^{M} \partial_{M}
$$


has a right action on superforms:

$$
\begin{aligned}
d \Omega_{(n)} & =\frac{1}{n !} d Z^{M_{n}} \wedge \ldots \wedge d Z^{M_{1}} \wedge d Z^{N} \partial_{N} \Omega_{M_{1} \ldots M_{n}} \\
& =\frac{1}{n !} E^{A_{n}} \wedge \cdots \wedge E^{A_{1}} \wedge E^{A}\left(D_{A} \Omega_{A_{1} \ldots A_{n}}+\frac{1}{2} n T_{A A_{1}}{ }^{B} \Omega_{B A_{2} \ldots A_{n}}\right), \\
d\left(\Omega_{(m)} \wedge \Omega_{(n)}\right) & =\Omega_{(m)} \wedge d \Omega_{(n)}+(-1)^{m} d \Omega_{(m)} \wedge \Omega_{(n)} .
\end{aligned}
$$

The right action of the interior product $i_{V}$, where $V$ is a supervector field, is

$$
\begin{aligned}
i_{V} \Omega_{(n)} & =\frac{1}{(n-1) !} E^{A_{n}} \wedge \cdots \wedge E^{A_{2}} V^{A} \Omega_{A A_{2} \ldots A_{n}}, \\
i_{V}\left(\Omega_{(m)} \wedge \Omega_{(n)}\right) & =\Omega_{(m)} \wedge i_{V} \Omega_{(n)}+(-1)^{m} i_{V} \Omega_{(m)} \wedge \Omega_{(n)} .
\end{aligned}
$$

\section{Appendix B. Proof of $\Gamma^{2}=\mathbb{1}$}

In this appendix we will prove that the matrix $\Gamma$ defined in (4.9) squares to $\mathbb{1}$. In order to simplify the notation somewhat we will only consider the type IIB case - the IIA case follows analogously.

For the type IIB D $p$-branes we can write (4.9) as

$$
d^{p+1} \xi \Gamma=\left.\frac{1}{\sqrt{-\operatorname{det}(g+\tilde{\mathscr{F}})}} e^{\tilde{\mathscr{F}}} \wedge X I\right|_{\mathrm{vol}},
$$

with

$$
X=\bigoplus_{n \in \mathbb{N}} \gamma_{(2 n)} K^{n}
$$

Hence, if we define $\tilde{\Gamma}=\sqrt{-\operatorname{det}(g+\tilde{\mathscr{F}})} \Gamma$, we have to show that $\tilde{\Gamma}^{2}=-\operatorname{det}(g+\tilde{\mathscr{F}})$. In dimension $p+1=2 m$ the square of $\tilde{\Gamma}$ is

$$
\begin{aligned}
\tilde{\Gamma}^{2}=-\varepsilon^{i_{1} \ldots i_{2 m}} \varepsilon^{j_{1} \ldots j_{2 m}} \sum_{k, r=0 ; k+r \text { even }}^{m}(-1)^{r} \frac{1}{(2 k) !(2 r) !} \gamma_{i_{1} \ldots i_{2 k}} \gamma_{j_{1} \ldots j_{2 r}} \\
\quad \times \frac{1}{(m-k) !(m-r) ! 2^{2 m-k-r}} \tilde{\mathscr{F}}_{i_{2 k+1} i_{2 k+2} \ldots \tilde{\mathscr{F}}_{i_{2 m-1} i_{2 m}} \tilde{\mathscr{F}}_{j_{2 r+1} j_{2 r+2}} \ldots \tilde{\mathscr{F}}_{j_{2 m-1} j_{2 m}},},
\end{aligned}
$$

where the overall sign is due to $I^{2}=-\mathbb{1}$, and the factor $(-1)^{r}$ comes from the fact that $I K^{r}=$ $(-1)^{r} K^{r} I$. Furthermore, due to the symmetry in the $i$ - and $j$-indices the sum $k+r$ will necessarily be even, implying that we have only even powers in $\tilde{\mathscr{F}}$. The $\gamma$-matrix products can be expanded as

$$
\gamma_{i_{1} \ldots i_{2 k}} \gamma_{j_{1} \ldots j_{2 r}}=\gamma_{i_{1} \ldots i_{2 k} j_{1} \ldots j_{2 r}}+\ldots+c_{q}^{k, r} g_{\left[j_{1} \ldots j_{2 q}\right.}^{\left[i_{1} \ldots i_{2 q}\right.} \gamma^{\left.i_{2 q+1} \ldots i_{2 k}\right]}{ }_{\left.j_{2 q+1} \ldots j_{2 r}\right]}+\ldots
$$


where

$$
\begin{aligned}
c_{q}^{k, r} & =(-1)^{q} \frac{(2 k) !(2 r) !}{(2 k-2 q) !(2 r-2 q) !(2 q) !}, \\
g_{j_{1} \ldots j_{2 q}}^{i_{1} \ldots i_{2 q}} & =g_{\left[i_{1}\left|j_{1}\right| \ldots g_{\left.i_{2 q}\right] j_{2 q}},\right.} \\
\gamma^{i_{2 q+1} \ldots i_{2 k} k}{ }_{j_{2 q+1} \ldots j_{2 r}} & =\gamma_{i_{2 q+1} \ldots i_{2 k} j_{2 q+1} \ldots j_{2 r}} .
\end{aligned}
$$

For the same reason as above only even powers of the metric appear in the expansion (B.4). Note that the super-script indices on $g$ and $\gamma$ have been introduced here only for notational reasons and should not be interpreted as having been raised by $g^{-1}$.

In order to get the factor $-\operatorname{det}(g+\tilde{\mathscr{F}})$ we sum over all terms in $\tilde{\Gamma}^{2}$ proportional to the identity matrix:

$$
\begin{aligned}
& \left.\tilde{\Gamma}^{2}\right|_{\mathbb{1}}=-\varepsilon^{i_{1} \ldots i_{2 m}} e^{j_{1} \ldots j_{2 m}} \sum_{k=0}^{m} \frac{1}{(2 k) !} g_{j_{1} \ldots j_{2 k}}^{i_{1} \ldots i_{2 k}} \frac{1}{(m-k) !(m-k) ! 2^{2 m-2 k}} \\
& \times \tilde{\mathscr{F}}_{i_{2 k+1} i_{2 k+2}} \ldots \tilde{\mathscr{F}}_{i_{2 m-1} i_{2 m}} \tilde{\mathscr{F}}_{j_{2 k+1} j_{2 k+2}} \ldots \tilde{\mathscr{F}}_{j_{2 m-1} j_{2 m}} .
\end{aligned}
$$

We move the indices between $\tilde{\mathscr{F}}$ 's using the identity $S_{\left[i_{1} \ldots i_{2 m+1}\right]}=0$. The result is schematically

$$
\varepsilon^{i} \varepsilon^{j}\left(g^{k}\right)_{i j}\left(\tilde{\mathscr{F}}^{m-k}\right)_{i i}\left(\tilde{\mathscr{F}}^{m-k}\right)_{j j}=\frac{2^{m-k}(m-k) !}{(2 m-2 k)-1) ! !} \varepsilon^{i} \varepsilon^{j}\left(g^{k}\right)_{i j}\left(\tilde{\mathscr{F}}^{2 m-2 k}\right)_{i j}
$$

which inserted in (B.6) gives

$$
\left.\tilde{\Gamma}^{2}\right|_{\mathbb{1}}=-\varepsilon^{i} \varepsilon^{j} \frac{1}{(2 m) !} \sum_{k=0}^{m}\left(\begin{array}{c}
2 m \\
2 k
\end{array}\right)\left(g^{k}\right)_{i j}\left(\tilde{\mathscr{F}}^{m-k}\right)_{i j} \equiv-\operatorname{det}(g+\tilde{\mathscr{F}})
$$

In order to complete the proof we must show that the terms in $\tilde{\Gamma}^{2}$ containing $\gamma$-matrices vanish. To this end, we return to the identity (B.4). Not all terms in this identity will survive; actually

$$
\varepsilon^{i} \varepsilon^{j} g_{\left[i_{1} \ldots i_{2 q}\right.}^{j_{1} \ldots j_{2 q}} \gamma_{\left.i_{2 q+1} \ldots i_{2 k} j_{2 q+1} \ldots j_{2 r}\right]}=0 \Rightarrow \varepsilon^{i} \varepsilon^{j} g_{\left[i_{1} \ldots i_{2 q}\right.}^{j_{1} \ldots j_{2 q}} \gamma_{\left.i_{2 q+1} \ldots i_{2 k}\right] j_{2 q+1} \ldots j_{2 r}}=0 .
$$

This places the constraint $2 q \geq 2(k+r-m)$ on $q$, which implies that if we look at a term of the power $2 s$ in $\tilde{\mathscr{F}}(\Rightarrow 2 q \geq 2 m-4 s)$ and the power $2 m-4 s+2 k$ in $g$, where $0 \leq k \leq s-1(k=s$ is excluded since this term is already accounted for in (B.6) above), we have the following sum to consider:

$$
\sum_{l=0}^{2 s-2 k} \alpha_{l}^{s, k}\left(g^{m-2 s+k}\right)_{i j} \gamma_{i^{(4 s-4 k-2 l)} j^{(2 l)}}\left(\tilde{\mathscr{F}}^{k+l}\right)_{i i}\left(\tilde{\mathscr{F}}^{2 s-k-l}\right)_{j j} .
$$

Here $\gamma_{i^{(r)} j^{(q)}}$ has $r i$-indices and $q j$-indices. From (B.5) we can read off the $\alpha_{l}^{s, k}$ coefficient

$$
\alpha_{l}^{s, k}=\frac{(-1)^{l}}{2^{2 s}(2 m-4 s+2 k) !(4 s-4 k-2 l) !(2 l) !(k+l) !(2 s-k-l) !} .
$$


Cederwall, von Gussich, Nilsson, Sundell, Westerberg: "The Dirichlet Super-p-Branes ...” $\ldots 18$

8

In the the sum (B.10) we move the $i$-indices in $\gamma$ over to $\tilde{\mathscr{F}}$. Using the relation

$$
\begin{aligned}
& \varepsilon^{i} \varepsilon^{j}\left(g^{m-2 s+k}\right)_{i j} \gamma_{i(4 s-4 k-2 l)} j^{(2 l)}\left(\tilde{\mathscr{F}}^{k+l}\right)_{i i}\left(\tilde{\mathscr{F}}^{2 s-k-l}\right)_{j j} \\
& \quad=\frac{2(2 s-k-j)}{2 j+1} \varepsilon^{i} \varepsilon^{j}\left(g^{m-2 s+k}\right)_{i j} \gamma_{i(4 s-4 k-2 l-1)} j^{(2 l+1)}\left(\tilde{\mathscr{F}}^{k+l}\right)_{i i}\left(\tilde{\mathscr{F}}^{2 s-k-l-1}\right)_{j j} \tilde{\mathscr{F}}_{i j}
\end{aligned}
$$

repeatedly in both directions, we obtain

$$
\begin{aligned}
& \varepsilon^{i} \varepsilon^{j}\left(g^{m-2 s+k}\right)_{i j} \gamma_{i(4 s-4 k-2 l)} j^{(2 l)}\left(\tilde{\mathscr{F}}^{k+l}\right)_{i i}\left(\tilde{\mathscr{F}}^{2 s-k-l}\right)_{j j} \\
& =\beta_{l}^{s, k} \varepsilon^{i} \varepsilon^{j}\left(g^{m-2 s+k}\right)_{i j} \gamma_{j(4 s-4 k)}\left(\tilde{\mathscr{F}}^{2 s-k}\right)_{i i}\left(\tilde{\mathscr{F}}^{k}\right)_{j j},
\end{aligned}
$$

where

$$
\begin{aligned}
& \beta_{l=0}^{s, k}=\beta_{l=2(s-k)}^{s, k}=1, \\
& \beta_{l}^{s, k}=\frac{(2 l-1) ! !(4 s-4 k-2 l-1) ! !(2 s-k-j) !(k+j) !}{(4 s-4 k-1) ! ! k !(2 s-k) !}, \quad l \neq 0,2(s-k) .
\end{aligned}
$$

Inserting these expressions in (B.10) we can factor out $g, \gamma$ and $\tilde{\mathscr{F}}$, leaving us with the sum $\sum_{l=0}^{2(s-k)} \alpha_{l}^{s, k} \beta_{l}^{s, k}$. Finally, by factoring out the $l$-independent part we find

$$
\sum_{l=0}^{2 s-2 k} \alpha_{l}^{s, k} \beta_{l}^{s, k}=A^{s, k} \sum_{l=0}^{2 s-2 k}(-1)^{l}\left(\begin{array}{c}
2 s-2 k \\
l
\end{array}\right)=0,
$$

thereby completing the proof.

\section{Appendix C. $\kappa$-VARiation of the DiraC-Born-Infeld ACTiON}

Below we give the details concerning the calculation of the $\kappa$-variation of the Dirac-Born-Infeld action that we left out in section 4. For pedagogical reasons we restrict the presentation to the type IIB branes, since this case displays all the relevant features. At the end of the appendix, we will indicate how the notation introduced in section 4 allows for a simultaneous analysis of the IIA and IIB cases.

Let us begin by recalling some results from section 4, written here specifically for the IIB case. When convenient, we will use the integer $m$ defined by $2 m=p+1$. The variation of the Dirac-Born-Infeld action with parameter $\Gamma \kappa$ is

$$
\delta_{\Gamma \kappa} I_{\mathrm{DBI}}=\int_{M} d^{p+1} \xi \mathscr{L}_{\mathrm{DBI}}\left(\frac{p-3}{4} \delta_{\Gamma \kappa} \phi+\frac{1}{2} \operatorname{tr}\left\{(g+\tilde{\mathscr{F}})^{-1}\left(\delta_{\Gamma \kappa} g+\delta_{\Gamma \kappa} \tilde{\mathscr{F}}\right)\right\}\right),
$$


where $\tilde{\mathscr{F}} \equiv e^{-\frac{\phi}{2}} \mathscr{F}$, and

$$
\begin{aligned}
\delta_{\Gamma \kappa} g_{i j} & =4 i\left(\bar{E}_{(i} \gamma_{j} \Gamma \kappa\right), \\
\delta_{\Gamma \kappa} \tilde{\mathscr{F}}_{i j} & =4 i\left(\bar{E}_{[i} \gamma_{j]} K \Gamma \kappa\right)+\left(\bar{\Lambda}\left(\gamma_{i j} K-\tilde{\mathscr{F}}_{i j}\right) \Gamma \kappa\right), \\
\delta_{\Gamma \kappa} \phi & =2 \bar{\Lambda} \Gamma \kappa .
\end{aligned}
$$

The expression (4.9) for $\Gamma$ reduces in the $\Pi$ B case to

$$
d^{p+1} \xi \Gamma=-\left.\frac{e^{\frac{1}{4}(p-3) \phi}}{\mathscr{L}_{\mathrm{DBI}}} e^{\tilde{\mathscr{F}}} \wedge X I\right|_{\mathrm{vol}}
$$

with

$$
X=\bigoplus_{n=0}^{m} \gamma_{(2 n)} K^{n}
$$

The variation (C.1) is naturally written as a sum $\delta_{\Gamma \kappa} I_{\mathrm{DBI}}=\Delta_{A}+\Delta_{B}+\Delta_{C}$. The first term corresponds to the first term on the right hand side of (C.1) and is the one that is easiest to calculate. Indeed, we only need to insert the expression for $\delta_{\Gamma \kappa} \phi$ from (C.2) in (C.1). We can then immediately use the form expansion (C.3) to obtain

$$
\begin{aligned}
\Delta_{A} & =-\frac{1}{2}(p-3) \int_{M} e^{\frac{1}{4}(p-3) \phi} e^{\tilde{F}} \wedge(\bar{\Lambda} X I \kappa) \\
& =-\frac{1}{2}(p-3) \int_{M} e^{\mathscr{F}} \wedge \bigoplus_{n=0}^{m} e^{\frac{1}{2}(n-2) \phi}\left(\bar{\Lambda} \gamma_{(2 n)} K^{n} I \kappa\right)
\end{aligned}
$$

In the last step we exploited the fact that only terms proportional to the volume form survive the integration, to move the dilaton factor in $\tilde{\mathscr{F}}$ over to the $\gamma$-terms. Note here that the total variation $\delta_{\Gamma \kappa} I_{\mathrm{DBI}}$ is not allowed to contain any $p$-dependent terms, if we are to be able to recover the supergravity constraints for the RR fields strengths by imposing $\delta_{\Gamma \kappa} I_{\mathrm{DBI}}+\delta_{\kappa} I_{\mathrm{Wz}}=0$. The $p$-dependence in (C.5) must therefore be canceled by the remaining terms $\Delta_{B}+\Delta_{C}$.

Having determined $\Delta_{A}$, we turn to $\Delta_{B}$ which we define as the $\Lambda$-independent part of $\delta_{\Gamma \kappa} I_{\mathrm{DBI}}$. This is the part that will eventually be canceled by the dimension-0-component field strength contribution to $\delta_{\kappa} I_{\mathrm{wz}}$. It is most conveniently calculated by first observing that

$$
\begin{aligned}
\Delta_{B} & \left.=2 i \int_{M} d^{2 m} \xi \mathscr{L}_{\mathrm{DBI}}\left((g+\tilde{\mathscr{F}})^{-1}\right)^{j i}\left(\bar{E}_{(i} \gamma_{j} \Gamma \kappa\right)+\left(\bar{E}_{[i} \gamma_{j]} K \Gamma \kappa\right)\right) \\
& =2 i \int_{M} d^{2 m} \xi \mathscr{L}_{\mathrm{DBI}}\left((g+\tilde{\mathscr{F}} K)^{-1}\right)^{j i}\left(\bar{E}_{i} \gamma_{j} \Gamma \kappa\right),
\end{aligned}
$$


due to the property $K^{2}=\mathbb{1}$. We then write $\left(\bar{E}_{i} \gamma_{j} \Gamma \kappa\right)=\left(\gamma_{j} \Gamma\right)_{\alpha \beta} E_{i}^{\alpha} \kappa^{\beta}$ and insert the expression (C.3) for $\Gamma$, thus finding

$$
\Delta_{B}=-2 i \int_{M} e^{\left(\frac{1}{2} m-1\right) \phi}\left((g+\tilde{\mathscr{F}} K)^{-1}\right)^{j i} \sum_{n=0}^{m}\left(\frac{1}{(m-n) !} \tilde{\mathscr{F}} m-n \wedge\left(\gamma_{j} \gamma_{(2 n)} K^{n} I\right)_{\alpha \beta}\right) E_{i}^{\alpha} \kappa^{\beta}
$$

By writing out the forms in components and using the $\gamma$-matrix identity

$$
\gamma_{j} \gamma_{i_{1} \ldots i_{2 n}}=\gamma_{j i_{1} \ldots i_{2 n}}+2 n g_{j\left[i_{1}\right.} \gamma_{\left.i_{2} \ldots i_{2 n}\right]}
$$

the sum in (C.7) above becomes

$$
\sum_{n=0}^{m}\left(\gamma_{j i_{1} \ldots i_{2 n}} \tilde{\mathscr{F}}_{i_{2 n+1} i_{2 n+2}}+\frac{2(n-k)}{2 k+1} g_{j i_{1}} \gamma_{i_{2} \ldots i_{2 n+2}} K\right) \frac{(\tilde{\mathscr{F}} m-n-1}{)_{i_{2 n+3} \ldots i_{2 m}} K^{n}} .
$$

Here we have left out the factor $d^{2 m} \xi \varepsilon^{i_{2 m} \ldots i_{1}}$ and relabeled the summation index in order to pair together terms with equal numbers of $\gamma$-matrices.

Cycling the $2 m+1$ indices on the left term using $S_{\left[j i_{1} \ldots i_{2 m}\right]}=0$ we can move the free index $j$ over to one of the $\tilde{\mathscr{F}}$ 's, at the same time picking up precisely the combinatorical factor that allows us to extract the matrix $(g+\tilde{\mathscr{F}} K)_{i_{1} j}$ (to achieve this we again need to use $K^{2}=\mathbb{1}$ ). This matrix is then contracted with its inverse appearing in (C.6) to yield $\delta_{i_{1}}^{i}$, and after some straightforward steps we arrive at the result

$$
\Delta_{B}=2 i \int_{M} e^{\mathscr{F}} \wedge \bigoplus_{n=1}^{m} e^{\frac{1}{2}(n-1) \phi}\left(\bar{E} \wedge \gamma_{(2 n-1)} K^{n} I \kappa\right)
$$

The third and final contribution to $\delta_{\Gamma \kappa} I_{\mathrm{DBI}}$ is the trickiest one to compute. It can be written as

$$
\Delta_{C}=\frac{1}{2} \int_{M} d^{2 m} \xi \mathscr{L}_{\mathrm{DBI}}\left((g+\tilde{\mathscr{F}})^{-1}\right)^{j i}\left(\left\{\left(\gamma_{i j} K+g_{i j}\right)-(g+\tilde{\mathscr{F}})_{i j}\right\} \Gamma\right)_{\alpha \beta} \Lambda^{\alpha} \kappa^{\beta} .
$$

Here we have added and subtracted $g_{i j}$, since we can then perform the trace in second term to obtain an expression of exactly the same form as $\Delta_{A}$, only with a different coefficient. By comparing these coefficients we can use (C.5) to immediately write down the result

$$
\Delta_{C_{2}}=\frac{1}{2}(p+1) \int_{M} e^{\mathscr{F}} \wedge \bigoplus_{n=0}^{m} e^{\frac{1}{2}(n-1) \phi}\left(\bar{\Lambda} \gamma_{(2 n)} K^{n} I \kappa\right)
$$

Note that the $p$-dependent part of $\Delta_{C_{2}}$ cancels the one of $\Delta_{A}$, leaving a contribution with formlevel-independent coefficient (apart from the dilaton factor). 
It remains to evaluate the contribution $\Delta_{C_{1}}$ from the term proportional to $\left(\gamma_{i j} K+g_{i j}\right)$ in (C.11). This is done by using essentially the same techniques as we did when determining $\Delta_{B}$. For $\Delta_{C_{1}}$, however, the manipulations are somewhat more intricate. In order not to lose the essential ideas behind a blur of coefficients and indices, we will be rather schematic, leaving to the interested reader to check explicitly that the signs and coefficients match as promised.

It is convenient to extract the matrix $T_{i j} \equiv\left(\gamma_{i j} K+g_{i j}\right) d^{2 m} \xi \mathscr{L}_{\mathrm{DBI}} \Gamma$ from (C.11) (here we suppress the spinor indices). Our strategy is as for $\Delta_{B}$ to try to rearrange the indices to obtain a factor $(g+\tilde{\mathscr{F}})_{i}$. that will cancel the inverse matrix in (C.11). In analogy with the treatment of $\Delta_{B}$ above we thus insert the expression (C.3), write the forms in components and expand the products of $\gamma$-matrices. For this case the relevant identity contains three terms on the right hand side:

$$
\gamma_{i j} \gamma_{i_{1} \ldots i_{2 n}}=\gamma_{i j i_{1} \ldots i_{2 n}}-4 n g_{\left[i\left|i_{1}\right|\right.} \gamma_{j] i_{2} \ldots i_{2 n}}-2 n(2 n-1) g_{\left[i\left|i_{1}\right|\right.} g_{j] i_{2}} \gamma_{i_{3} \ldots i_{2 n}}
$$

(recall that there is a factor $\varepsilon^{i_{2 m} \ldots i_{1}}$ in $T_{i j}$ enforcing antisymmetrization in the $2 m$ indices $i_{1} \ldots i_{2 m}$ ). After the proper relabeling of the summation index $n$ for the first and third term, $T_{i j}$ can be written as sum of terms homogeneous in powers of $\gamma$-matrices. Schematically we thus have

$$
T_{i j} \sim \sum_{n=0}^{m}\left(\left\{\gamma_{i j} \tilde{\mathscr{F}}^{m-n+1}+g_{i} g_{j} \gamma \tilde{\mathscr{F}}^{m-n-1}+g_{i j} \tilde{\mathscr{F}}^{m-n}\right\} K^{n}+g_{[i} \gamma_{j]} \tilde{\mathscr{F}}^{m-n} K^{n+1}\right)
$$

Here and in the sequel we leave out the exact coefficients of the respective terms as well as the contracted indices $i_{1} \ldots i_{2 m}$. Furthermore, all $\gamma$ 's have $2 n$ indices and we have used the property $K^{2}=\mathbb{1 1}$.

The "boundary terms" of the sum will need some special consideration, but since this presents no additional complications we will not give the details here. As an amusing example of how analysing boundary terms can reveal useful information, it is worth mentioning, however, that if one considers $\Delta_{C}$ as a whole using instead of (4.15) the identity

$$
\delta \operatorname{det} M=\frac{1}{(n-1) !} \varepsilon^{i_{1} \ldots i_{n}} \varepsilon^{j_{1} \ldots j_{n}} M_{i_{1} j_{1} \ldots M_{i_{n-1} j_{n-1}}} \delta M_{i_{n} j_{n}}
$$

one finds after expanding the $\gamma$-matrix products that the $\tilde{\mathscr{F}}$-independent terms in the formexpansion sum must vanish for symmetry reasons. This means that for fixed $p$ the $\mathscr{F}$-independent term in (4.14) containing the dimension- $\frac{1}{2}$-component of $R_{(p+2)}$ is canceled solely by the $\mathscr{F}$ independent term in $\Delta_{A}$ which was very straightforward to determine. Hence we can immediately read off the components $R_{a_{1} \ldots a_{p+1} \alpha}$ from (C.5) without having to calculate the more difficult term $\Delta_{C}$. Of course, in order to prove $\kappa$-invariance we have to go through the whole calculation, but this observation provides a very strong hint that we are on the right track. 
After this short excursion, let us thus return to the computation of $\Delta_{C_{1}}$. We use again the trick of antisymmetrizing in $2 m+1$ indices to reposition the indices $i$ and $j$. For the first term in (C.14) one obtains in this way

$$
\gamma_{i j} \tilde{\mathscr{F}}^{m-n+1} \sim \tilde{\mathscr{F}}_{[i} \gamma_{j]} \tilde{\mathscr{F}}^{m-n}=\tilde{\mathscr{F}}_{i} \gamma_{j} \tilde{\mathscr{F}}^{m-n},
$$

where in the last step we used the observation that the symmetrized piece vanishes by cycling the indices $j i_{1} \ldots i_{2 m}$. The term $(\mathrm{C} .16)$ is the $\tilde{\mathscr{F}}$-part of the matrix $(g+\tilde{\mathscr{F}})_{i}$. that we wish to extract from $T_{i j}$. It thus remains to find the corresponding $g$-term and show that the rest vanishes.

The required term can be isolated from the third term in (C.14); after cycling the indices $j i_{1} \ldots i_{2 m}$ the latter reads

$$
g_{i j} \tilde{\mathscr{F}}^{m-n} \sim g_{i} \gamma_{j} \tilde{\mathscr{F}}^{m-n}-g_{[i} \gamma_{j]} \tilde{\mathscr{F}}^{m-n}+g_{(i} \tilde{\mathscr{F}}_{j)} \gamma^{\tilde{\mathscr{F}}^{m-n-1}} .
$$

The first term on the right hand side is exactly the one we want; together with (C.16) it gives

$$
\Delta_{C_{1}}=-\int_{M} e^{\mathscr{F}} \wedge \bigoplus_{n=0}^{m} n e^{\frac{1}{2}(n-1)}\left(\bar{\Lambda} \gamma_{(2 n)} K^{n} I \kappa\right)
$$

Here we have anticipated that the remaining terms

$$
\begin{aligned}
\tilde{T}_{i j} \sim \sum_{n=0}^{m}\left(\left\{g_{i} g_{j} \gamma \tilde{\mathscr{F}}^{m-n-1}\right.\right. & \left.+g_{[i} \gamma_{j]} \tilde{\mathscr{F}}^{m-n}+g_{(i} \tilde{\mathscr{F}}_{j)} \gamma \tilde{\mathscr{F}}^{m-n-1}\right\} K^{n} \\
& \left.+g_{[i} \gamma_{j]} \tilde{\mathscr{F}}^{m-n} K^{n+1}\right)
\end{aligned}
$$

in $T_{i j}$ will cancel out; we will now show that this is really the case.

We begin by noticing that the last term has a different $K$-dependence, and must vanish by itself. Indeed, by cycling again in $j i_{1} \ldots i_{2 m}$ the index $j$ ends up only on an $\tilde{\mathscr{F}}$-factor due to the symmetry of $g$. By contracting the resulting $g_{[i} \tilde{\mathscr{F}}_{j]}$ with the matrix $\left((g+\tilde{\mathscr{F}})^{-1}\right)^{j i}$ one finds after some straightforward algebra that the term is proportional to the matrix

$$
\tilde{\mathscr{F}}\left(1+g^{-1} \tilde{\mathscr{F}}\right)^{-1}-\left(1+\tilde{\mathscr{F}} g^{-1}\right)^{-1} \tilde{\mathscr{F}}
$$

which is readily found to vanish identically.

The cancellation of the remaining three terms in (C.19) is a bit less obvious; by cycling the indices $j i_{1} \ldots i_{2 m}$ in the second term we obtain a term $g_{[i} \tilde{\mathscr{F}}_{j]} \gamma \tilde{\mathscr{F}}^{m-n-1}$ with the right coefficient to combined with the third term to give the term $g_{i} \tilde{\mathscr{F}}_{j} \gamma \tilde{\mathscr{F}}^{m-n-1}$ containing no explicit (anti)symmetrization. When added to the first term in (C.19) the latter gives us the term 
$g_{i}(g+\tilde{\mathscr{F}})_{. j}$, which can be contracted with $\left((g+\tilde{\mathscr{F}})^{-1}\right)^{j i}$ from the left. The result is a factor $g_{i_{1} i_{2}}$ which vanishes due to the antisymmetrization in the indices $i_{1} \ldots i_{2 m}$. This concludes the proof of (C.18).

Let us then collect the results that we have obtained; combining (C.5), (C.10), (C.12) and (C.18) we find the following expression for the $\kappa$-variation of the Dirac-Born-Infeld action for a general IIB D $p$-brane:

$$
\begin{aligned}
\delta_{\Gamma \kappa} I_{\mathrm{DBI}}=\int_{M} e^{\mathscr{F}} \wedge \bigoplus_{n}[ & 2 i e^{\frac{1}{2}(n-1) \phi}\left(\bar{E} \wedge \gamma_{(2 n-1)} K^{n} I \kappa\right) \\
& \left.+(2-n) e^{\frac{1}{2}(n-1) \phi}\left(\bar{\Lambda} \gamma_{(2 n)} K^{n} I \kappa\right)\right] .
\end{aligned}
$$

As mentioned in the beginning of this appendix, the above calculations can be performed quite analogously for the $\Pi \mathrm{A}$ case, or even simultaneously for the all $\mathrm{D} p$-branes. The only essential difference between the ПА and ПВ cases, as far as $\kappa$-symmetry is concerned, can be traced to the fact that $\gamma_{11}$ anticommutes whereas $K$ commutes with the $\gamma$-matrices. The notation introduced in section 4 has been tailored specifically to deal with the consequences of this difference in an efficient manner.

A convenient starting-point for a simultaneous treatment of the $\Pi \mathrm{A}$ and $\Pi \mathrm{B}$ D-branes is the following expression for the $\kappa$-variations listed in (4.17):

$$
\begin{aligned}
d^{p+1} \xi \delta_{\Gamma \kappa}(g+\tilde{\mathscr{F}})_{i j} & =-\frac{e^{\frac{p-3}{4} \phi}}{\mathscr{L}_{\mathrm{DBI}}} e^{\tilde{\mathscr{F}}} \wedge\left(4 i \bar{E}_{(i} \gamma_{j} X Y \kappa+4 i \bar{E}_{[i} \gamma_{j]} X P Y \kappa\right. \\
& \left.+\bar{\Lambda} \gamma_{i j} X P Y \kappa-\bar{\Lambda} X Y \kappa \tilde{\mathscr{F}}_{i j}\right) .
\end{aligned}
$$

Note that for type $\Pi$ A we have $X P=-P X$. It is however only when the combination $X P$ is used in (C.22) that the type ПA expression is formally equivalent (signwise) to the type ПB expression, since $X$ is always written with $P$ to the right in (4.9). This observation is of course irrelevant for the final answer, but relevant if one wishes to treat type $\Pi A$ and type $\Pi B$ simultaneously. The outcome of such an analysis is the expressions (4.18) and (4.19). 


\section{REFERENCES}

[1] M. Cederwall, A. von Gussich, B.E.W. Nilsson and A. Westerberg,

"The Dirichlet super-three-brane in type IIB supergravity", hep-th/9610148.

[2] M. Aganagic, C. Popescu and J.H. Schwarz, "D-brane actions with local kappa symmetry", hep-th/9610249.

[3] J. Polchinski, "Dirichlet-branes and Ramond-Ramond charges", Phys. Rev. Lett. 75 (1995) 4724 (hep-th/9510017.

[4] J. Polchinski and E. Witten, "Evidence for heterotic-type I string duality", Nucl. Phys. B460 (1996) 525 (hep-th/9510169).

[5] E. Witten, "Small instantons in string theory", Nucl. Phys. B460 (1996) 541 hep-th/9511030.

[6] E. Bergshoeff, E. Sezgin and P.K. Townsend, "Supermembranes and eleven-dimensional supergravity", Phys. Lett. B189 (1987) 75;

"Properties of the eleven-dimensional supermembrane theory", Ann. Phys. 185 (1988) 330.

[7] A. Achúcarro, J.M. Evans, P.K. Townsend and D.L. Wiltshire, "Super p-branes", Phys. Lett. B198 (1987) 441.

[8] E. Bergshoeff, E. Sezgin and P.K. Townsend, "Super p-branes as gauge theories of volume-preserving diffeomorphisms", Ann. Phys. 199 (1990) 340.

[9] M.J. Duff and J.X. Lu, "Type II p-branes: the brane-scan revisited", Nucl. Phys. B390 (1993) 276 (hep-th/9207060).

[10] P.S. Howe and E. Sezgin, "Superbranes", hep-th/9607227.

[11] E. Witten, "String theory dynamics in various dimensions", Nucl. Phys. B443 (1995) 85 (hep-th/9503124).

[12] J.H. Schwarz, "The power of M theory", Phys. Lett. B367 (1996) 97 (hep-th/9510086); "Lectures on superstring and $M$ theory dualities", hep-th/9607201.

[13] A. Sen, "Unification of string dualities", hep-th/9609176.

[14] E. Sezgin, "The M algebra", hep-th/9609086.

[15] T. Banks, W. Fischler, S.H. Shenker and L. Susskind, "M theory as a matrix model: a conjecture", hep-th/9610043.

[16] L. Brink and P. Howe, "Eleven-dimensional supergravity on the mass-shell in superspace", Phys. Lett. 91B (1980) 384 .

[17] E. Cremmer and S. Ferrara, "Formulation of eleven-dimensional supergravity in superspace", Phys. Lett. 91B (1980) 61.

[18] R. Güven, "Black p-brane solutions of D=11 supergravity theory", Phys. Lett. B276 (1992) 49.

[19] M.J. Duff and K.S. Stelle, "Multimembrane solutions of D=11 supergravity" Phys. Lett. B253 (1991) 113.

[20] C.M. Hull and P.K. Townsend, "Unity of superstring dualities", Nucl. Phys. B438 (1995) 109 (hep-th/9410167).

[21] C.M. Hull, "String dynamics at strong coupling", Nucl. Phys. B468 (1996) 113 hep-th/9512181).

[22] P.K. Townsend, "p-brane democracy", hep-th/9507048.

[23] E. Bergshoeff, C.M. Hull and T. Ortín, "Duality in the type-II superstring effective action", Nucl. Phys. B452 (1995) 547 hep-th/9504081).

[24] M.J. Duff, R.R. Khuri and J.X. Lu, "String solitons", Phys. Rep. 259 (1995) 213 (hep-th/9412184).

[25] M.J. Duff, "Strong/weak coupling duality from the dual string", Nucl. Phys. B442 (1995) 47 hep-th/9501030.

[26] K. Behrndt, E. Bergshoeff and B. Jansen, "Type II duality in six dimensions", Nucl. Phys. B467 (1996) 100 hep-th/9512152).

[27] O. Aharony, "String theory dualities from M theory", Nucl. Phys. B476 (1996) 470 (hep-th/9604103.

[28] M. Berkooz, R.G. Leigh, J. Polchinski, J. H. Schwarz, N. Seiberg and E. Witten, "Anomalies, dualities, and topology of $D=6 N=1$ superstring vacua", Nucl. Phys. B475 (1996) 115 (hep-th/9605184).

[29] R.G. Leigh, "Dirac-Born-Infeld action from Dirichlet sigma model", Mod. Phys. Lett. A4 (1989) 2767. 
Cederwall, von Gussich, Nilsson, Sundell, Westerberg: “The Dirichlet Super-p-Branes . . . $\ldots 25$

[30] A.A. Tseytlin, "Self-duality of Born-Infeld action and Dirichlet 3-brane of Type IIB superstring", Nucl. Phys. B469 (1996) $5^{1}$ (hep-th/9602064).

[31] M.B. Green and M. Gutperle, "Comments on 3-branes", Phys. Lett. B377 (1996) 28 (hep-th/9602077).

[32] M. Douglas, "Branes within branes", hep-th/9512077.

[33] M.B. Green, C.M. Hull and P.K. Townsend,

"D-brane Wess-Zumino actions, T-duality and the cosmological constant", hep-th/9604119.

[34] P.S. Howe and P.C. West, "The complete N=2, $d=10$ supergravity", Nucl. Phys. B238 (1984) 181.

[35] J.L. Carr, S.J. Gates Jr. and R.N. Oerter, " $D=10, N=2 A$ supergravity in superspace", Phys. Lett. 189B (1987) 68.

[36] P.K. Townsend, "D-branes from M-branes", Phys. Lett. B373 (1996) 68 (hep-th/9512062).

[37] C. Schmidhuber, "D-brane actions", Nucl. Phys. B467 (1996) 146 (hep-th/9601003).

[38] M. Cederwall, A. von Gussich, A. Miković, B.E.W. Nilsson and A. Westerberg, "On the Dirac-Born-Infeld action for D-branes", Phys. Lett. B (to appear), hep-th/9606173, .

[39] A. Candiello and K. Lechner, "Duality in supergravity theories", Nucl. Phys. B412 (1994) 479 (hep-th/9309143).

[40] P.S. Howe and E. Sezgin, " $d=11, p=5 "$, hep-th/9611008. 